\title{
Etude de la distribution des phases nitrures dans le cas de la nitruration du fe-cr.
}

\author{
N.BENRABIA1, M.E.DJEGHLAL2, L.BARRALIER3. \\ 1 Département de Métallurgie, L'USTO. \\ BP 1505 Mnaour, Oran, Algérie. E-mail: Nacerbenrabia@yahoo.fr \\ 2 Département de Métallurgie, Ecole Nationale Polytechnique. \\ 10, Avenue Pasteur -BP N ${ }^{\circ}$ 182- El-Harrach Alger, Algérie. \\ 3 Laboratoire Méca-Surf, E.N.S.A.M, 02 Cours des Arts et Métiers, \\ 13617, Aix-en-Provence, Cedex 1, France.
}

\begin{abstract}
RESUME :
Du Fe-Cr (1 à $5 \%$ de chrome) a été élaboré puis nitruré dans des conditions industrielles c.-à-d $\left(520^{\circ} \mathrm{C}\right)$. La caractérisation par DRX a permis de tracer les profils des phases nitrures. Des calculs thermodynamiques d'équilibre de phase par le biais du logiciel ThermoCalc ont permis de tracer des courbes de profil des phases en fonction de l'azote et du chrome, et d'objectiver des courbes de teneur des phases en chrome et/ou en azote en fonction des éléments contenus dans l'alliage (azote et chrome). Des conclusions ont été tirées sur l'influence de l'azote et du chrome l'un sur l'autre et sur les phases nitrures.
\end{abstract}

Mots clés :

Nitruration, Thermocalc, équilibre de phases

\section{INTRODUCTION :}

La nitruration consiste à enrichir la surface d'un alliage en azote jusqu'à saturation. L'azote apporté va réagir avec les éléments constituant l'alliage pour donner des nitrures de ces éléments ou des nitrures de la combinaison de ces éléments.

Dans le cas de la nitruration des aciers CrMoV on obtient trois principaux nitrures, $\varepsilon$, $\gamma^{\prime}$ et $\alpha$. Ce dernier nitrure $(\alpha)$ ou la nitroferrite constitue le siège de diverses interactions entre l'azote et les éléments d'alliage contenus dans cette phase, ce qui va donner lieu à la formation de nouveaux nitrures qui vont influer sur les propriétés mécaniques du produit. Parmi ces nitrures on citera le nitrure de chrome CrN largement rencontré dans ce type de traitement.

L'apparition et l'abondance de ce nitrure va être décisive sur la consolidation des propriétés mécanique de la pièce traitée. Rappelons que les nitrures $\varepsilon$ ou $\gamma^{\prime}$ sont des éléments qui renforcent les propriétés d'usure et de dureté de l'alliage, alors que le nitrure $\alpha$ avec tout les précipités qu'il peut contenir renforce les propriétés d'endurance de l'alliage par la genèse des contraintes résiduelles de compression. Le nitrure $\mathrm{CrN}$ a une grande contribution dans la tenue à la fatigue de ces alliages, par ailleurs son taux sa distribution et sa forme ont une influence sur le résultat de la consolidation mécanique obtenu après nitruration.

Cette étude traite le système $\mathrm{Fe}-\mathrm{Cr}$ et met en évidence les paramètres qui favorisent la formation des différents nitrures. Une étude thermodynamique complémentaire a été introduite dans le but d'apporter plus d'informations sur le système étudié concernant le profil des phases et la distribution des éléments entre les phases, sachant que le dosage des éléments dans les phases reste difficilement accessible par l'expérimental dans le plupart des cas.

\section{ETUDE EXPERIMENTALE}

\subsection{Préparation des échantillons}

Un mélange de fer et du chrome pur en poudre ont été compressés sous forme de petites pastilles puis introduits dans un four à induction pour être fondus.

De cette fusion on a obtenu des petites pastilles d'un alliage binaire $\mathrm{Fe}-\mathrm{Cr}$ avec diverses composition (1,2,3,4 et $5 \% \mathrm{Cr}$ ) homogènes dans tout leurs volumes. Ces compositions ont été choisies pour expliquer le comportement du chrome dans les aciers CrMoV, dont la concentration en chrome est autours de ces valeurs. Les pastilles ont été par la suite trempées à $930^{\circ} \mathrm{C}$ et revenues à $600^{\circ} \mathrm{C}$ (ce qui correspond à une température juste au dessus de la température de nitruration).

Par la suite les échantillons ont été introduits dans un four de nitruration gazeuse, où ils ont été nitrurés à $520^{\circ} \mathrm{C}$ pendant 70 heures avec un taux de décomposition de l'ammoniaque de $15 \%$.

\subsection{Analyses de composition}

Avant d'être analysés les échantillons ont subi un polissage électrolytique de 0 à $300 \mu \mathrm{m}$ avec une vitesse d'érosion de $4-5 \mu \mathrm{m} / \mathrm{min}$.

Nous avons exposé ces échantillons aux rayons $X$ produits par une cathode en cobalt ce qui a permis l'obtention des diffractogrammes représentés ci-dessous (Figure 1).

This is an Open Access article distributed under the terms of the Creative Commons Attribution-Noncommercial License (http://creativecommons.org/licenses/by-nc/3.0/), which permits unrestricted use, distribution, and reproduction in any noncommercial medium, provided the original work is properly cited. 


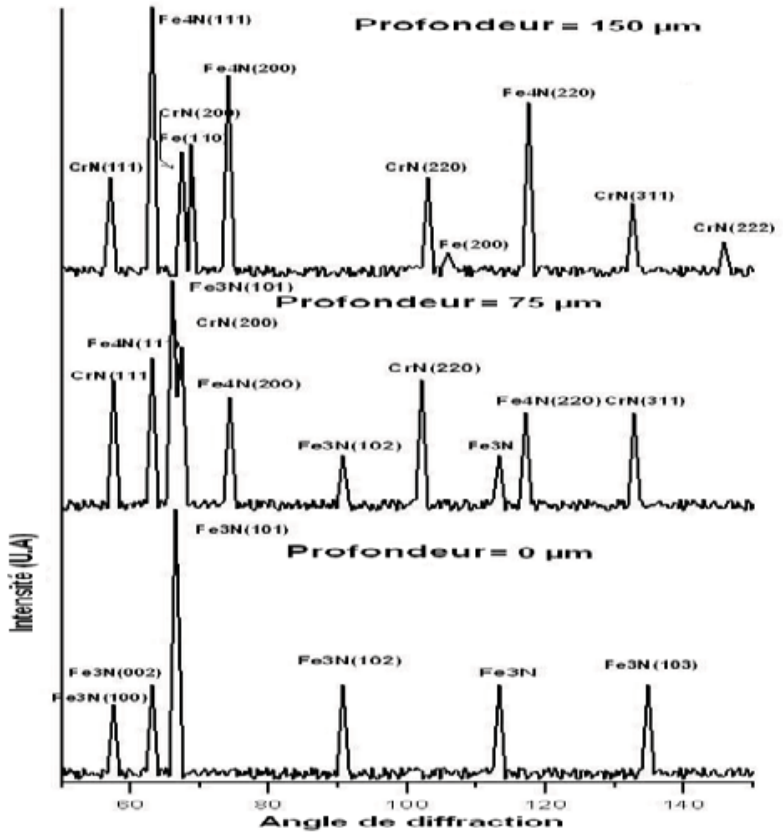

Figure 1. Diffractogrammes du binaire $\mathrm{Fe}-\mathrm{Cr}, 3 \% \mathrm{Cr}$.

\subsection{Distribution de phases}

En utilisant les diagrammes de diffractions des alliages nous avons calculé la composition surfacique de phase pour chaque profondeur et ce par la méthode de comparaison des intensités. Vue l'isotropie des alliages étudiés les résultats peuvent être généralisés à la composition volumique. Le résultat est représenté sur les figure $(2,3,4)$ ci-dessous.

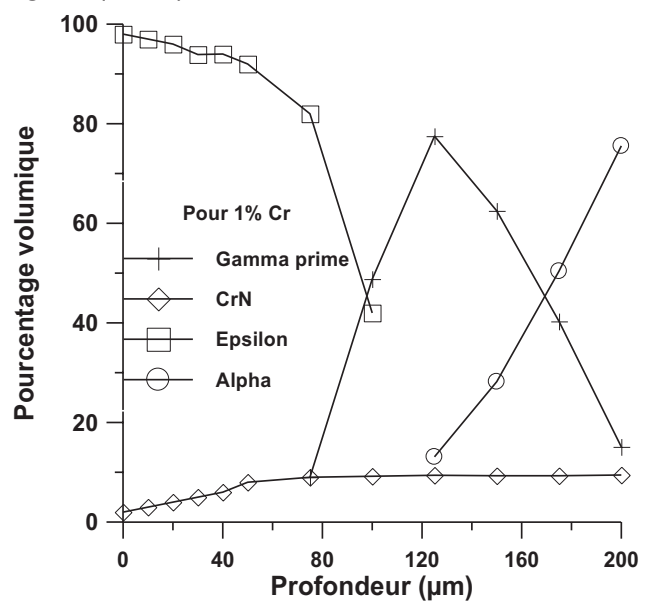

Figure 2. Fraction volumique des phases pour $1 \% \mathrm{Cr}$.

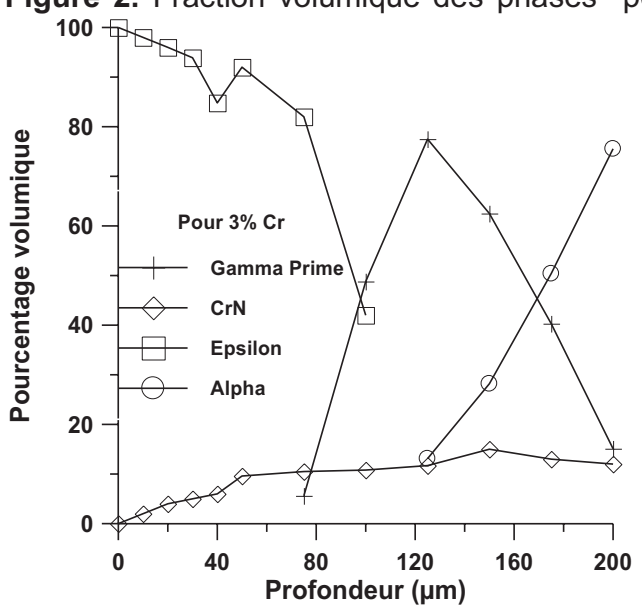

Figure 3. Fraction volumique des phases pour $3 \% \mathrm{Cr}$.

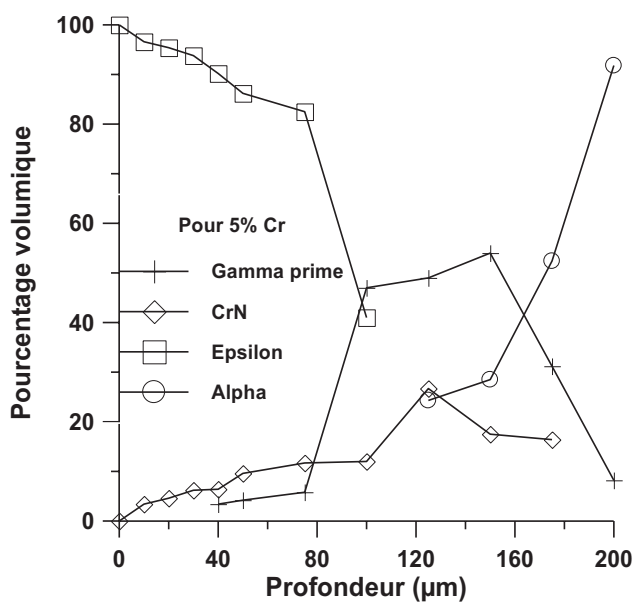

Figure 4. Fraction volumique des phases pour $5 \% \mathrm{Cr}$.

\subsection{Commentaires}

On peut voir à travers ces résultats que $\varepsilon$ est la phase la plus externe pour tout les alliages utilisés comme il a été montré par Barralis [1] et Leroy [2], $\gamma^{\prime}$ la phase médiane, et ces deux phases forment la couche de combinaison (la couche blanche) rencontrée dans la bibliographie $[1,2,3]$, la phase la plus profonde est la phase $\alpha$.

$\varepsilon$ la phase la plus riche en azote (28.2 à 32 at $\% \mathrm{~N}$ ) [2] se distingue jusqu'à une profondeur de 40 à $80 \mu \mathrm{m}, \gamma^{\prime}(19.3$ à 20.8 at $\%$ N) va jusqu'à 120 à $160 \mu \mathrm{m}$. Ces deux phases se trouvent combinées dans un domaine de 20 à $60 \mu \mathrm{m}$. Elles ne sont pas complètement distinctes comme dans le cas du fer nitruré, Torchane [4] et Somers [5], ni complètement combinées comme dans cas de la nitruration de l'acier selon Somers [6] et Mridha [7].

Le nitrure $\mathrm{CrN}$ se présente avec une forte concentration dans les couches médianes autours de l'interface $\alpha / \gamma$ ' (60 à $160 \mu \mathrm{m})$. Dans cette zone la teneur en azote est suffisante pour former ce nitrure alors que les phases voisines ne nécessitent pas un taux d'azote élevé. A noté que la quantité du nitrure $\mathrm{CrN}$ n'est pas très importante au-dessous de $3 \% \mathrm{Cr}$ à cause de la basse teneur de ces alliages en chrome. Ceci va donner des nitrures fins et dispersés que la température de nitruration ne permet pas leurs croissances, ce qui les rend difficilement décelables. La fraction de $\mathrm{CrN}$ croit avec la teneur en chrome de l'alliage.

L'augmentation de la quantité de chrome réduit la profondeur des différentes couches rencontrées dans un alliage nitruré. Comme il a été montré par Pope [8] pour le Fe-V et Driver [9] pour le Fe-Mo, des nitrures de vanadium et de molybdène apparaissent dans la zone nitrurée, ce qui produit une barrière à la diffusion de l'azote de la surface vers le cœur empêchant ainsi la croissance des couches nitrures et par conséquent réduit l'épaisseur des couches nitrures, il comprime la zone nitrurée et la présente comme une combinaison de divers nitrures, c'est le cas des aciers nitrurés[7,8].

A noter que le profil expérimental des éléments n'a pas été effectué dans cette étude à cause de la difficulté de doser les éléments dans les phases plus précisément l'azote qui nécessite l'emploi de l'EDAX ou EDX dont on ne disposait pas.

\section{CALCULS THERMODYNAMIQUES :}

\subsection{Les phases étudiées :}


Des calculs sur le système $\mathrm{Fe}-\mathrm{Cr}-\mathrm{N}$ ont été effectués par le logiciel de calculs thermodynamique ThermoCalc en adoptant des paramètres de calcul qui avoisinent les conditions pratiques de traitement de nos alliages.

Les phases mises à l'étude sont toutes les phases susceptibles d'exister dans de tels alliages, elles sont résumées dans le tableau ci-dessous :

\begin{tabular}{|l|l|l|}
\hline Phase & Structure & Formule chimique \\
\hline Alpha & BCC_A2 & (Fe,Cr)3N \\
\hline Gamma & FCC_A1\#1 & (Fe,Cr)N \\
\hline Gamma Prime & Fe4N & Fe4N \\
\hline Epsilon & HCP_A3\#1 & Fe2-3N \\
\hline CrN & FCC_A1\#2 & CrN \\
\hline Cr2N & HCP_A3 \#2 & Cr2N \\
\hline
\end{tabular}

Tableau 1. Les phases nitrures étudiées.

3.2. Influence des éléments chrome et azote sur le profil des phases:

Des calculs ont été développés par la méthode du «Steping» pour mettre en évidence la distribution des phases en fonction des conditions opératoires. Les données ont été tirées de la base de données SSOL2. On a opté pour l'utilisation de cette base parce qu'elle couvre bien le système étudié ( $\mathrm{Fe}-\mathrm{Cr}-\mathrm{N}$ ) et permet une future extension de calculs pour des systèmes plus complexes (cas des aciers). La base de données des aciers ne peut être directement utilisée parce que nos alliages ne contiennent pas de carbone.

Après calcul nous avons obtenu le résultat des figures cidessous.

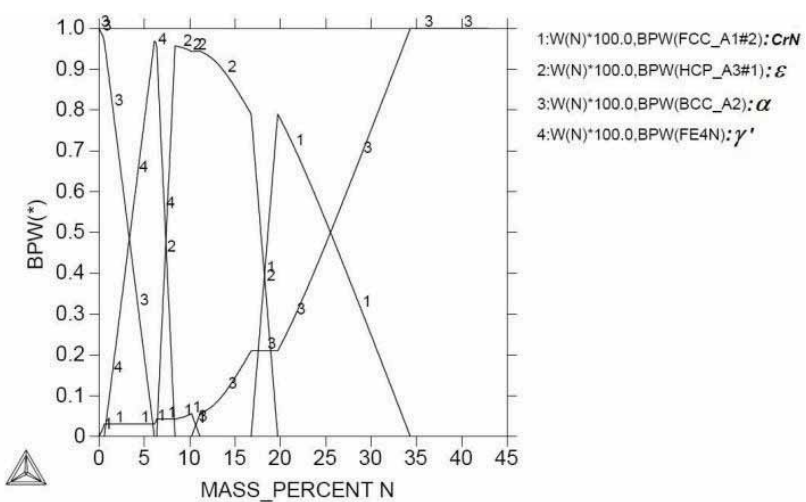

Figure 5. Pourcentage de phase en fonction de l'azote pour $1 \%$ de chrome.

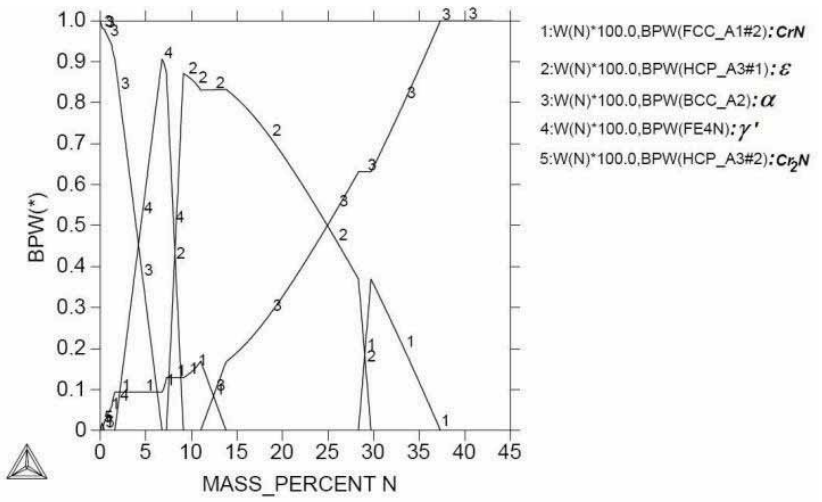

Figure 6. Pourcentage de phase en fonction d l'azote pour $3 \%$ de chrome.

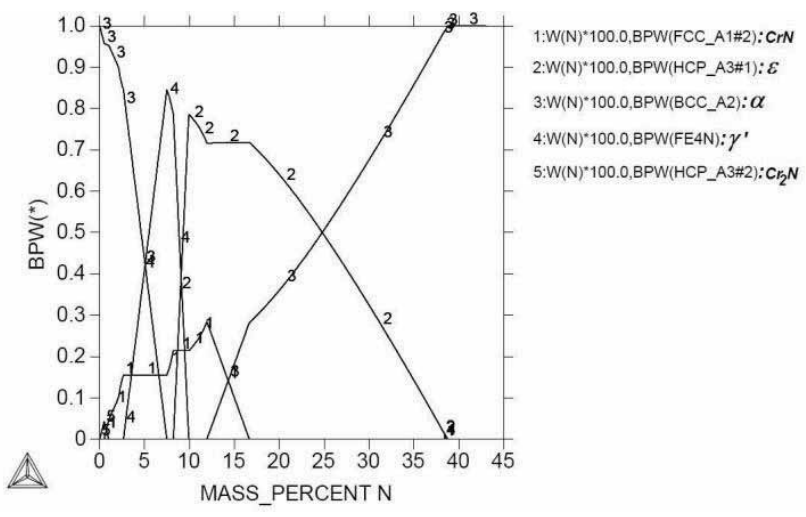

Figure 7. Pourcentage de phase en fonction d l'azote pour $5 \%$ de chrome.

\subsection{Teneur des phases en éléments d'alliages :}

L'identification des phases a été effectuée à partir des profils de distribution des éléments d'alliage (le chrome et l'azote). Des exemples sont exposés dans la figure ci-dessous où sont portés en ordonnées les pourcentages de chrome (ou d'azote) dans les phases en fonction du pourcentage de chrome total, ou d'azote total.

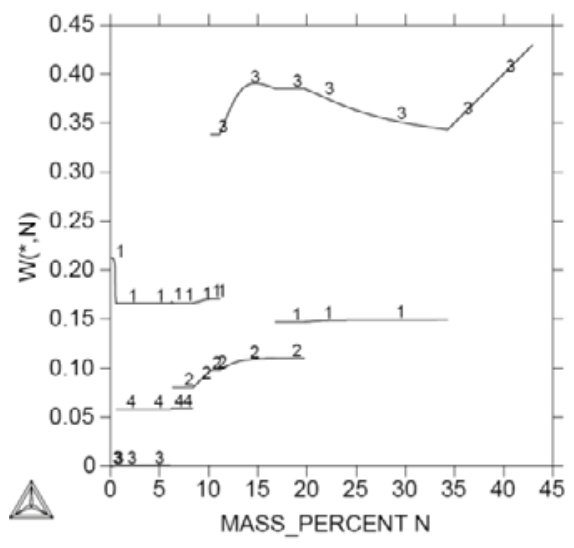

WW(N)*100.0,W(FCC_A1\#2,N 3:W(N) $100.0 . W(B C C-A 2 . N$

Figure 8. Teneurs en azote des phases en fonction du pourcentage d'azote dans l'alliage à $1 \% \mathrm{Cr}$.

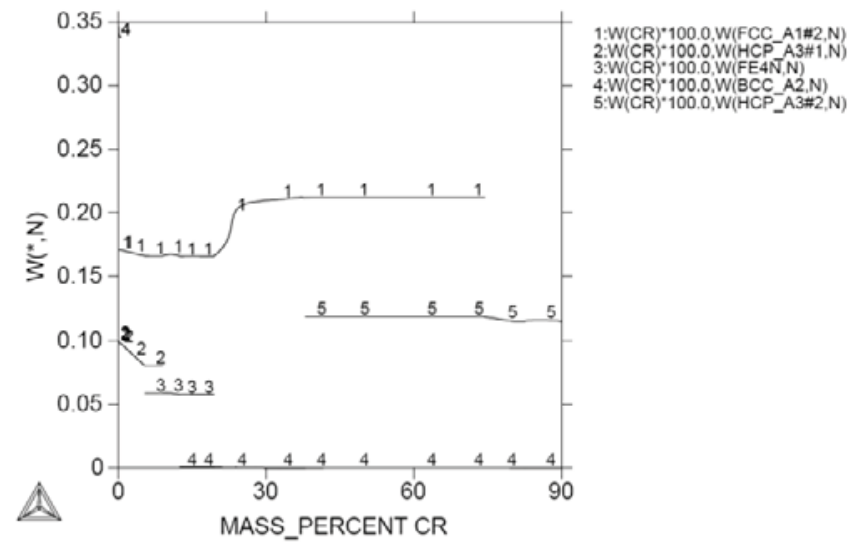

Figure 9. Teneurs en azote des phases en fonction du pourcentage de chrome dans l'alliage. 


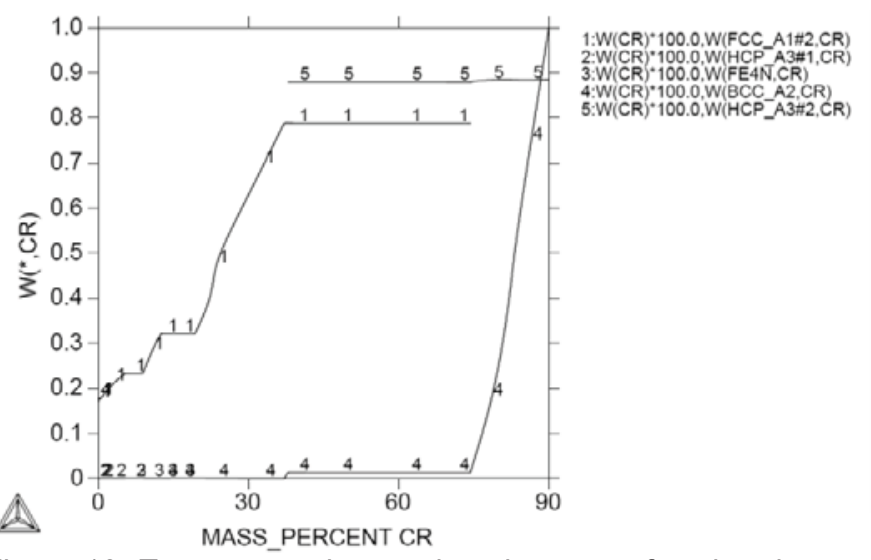

Figure 10. Teneurs en chrome des phases en fonction du pourcentage de chrome dans.

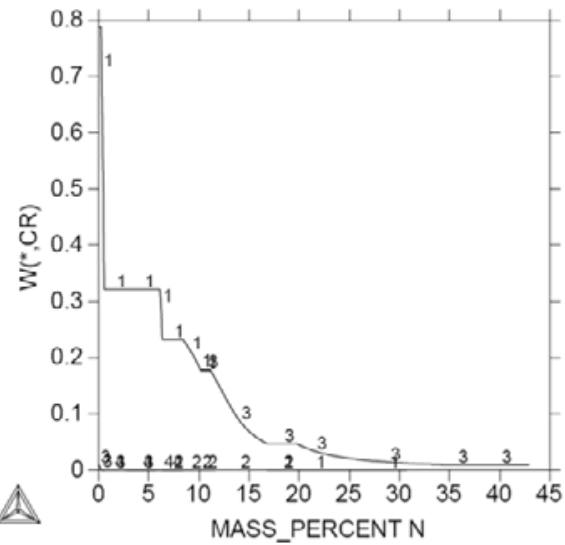

Figure 11. Teneurs en chrome des phases en fonction du pourcentage d'azote dans l'alliage à $1 \% \mathrm{Cr}$.

\subsection{Influence de la température sur le profil des} phases :

Nous avons constaté que les résultats des calculs correspondent bien à ce qui a été observé dans nos propres résultats expérimentaux, nous avons tenu à extrapoler ces calculs à des températures avoisinant les $520^{\circ} \mathrm{C}$, afin de voir l'influence de la température sur la distribution des phases nitrures obtenues.

A titre d'exemple nos exposons les figures ci-dessous.

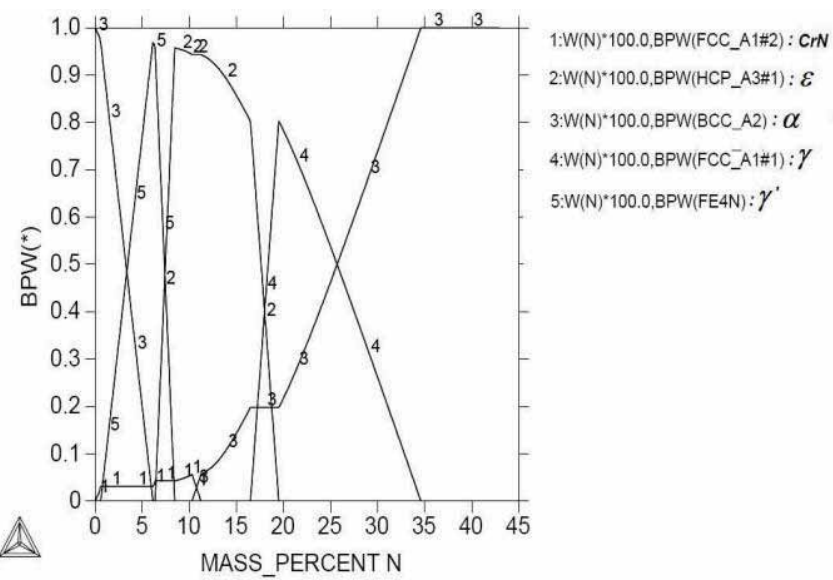

Figure 12. Profil de distribution des phases à $510^{\circ} \mathrm{C}$ et $1 \%$ Cr.

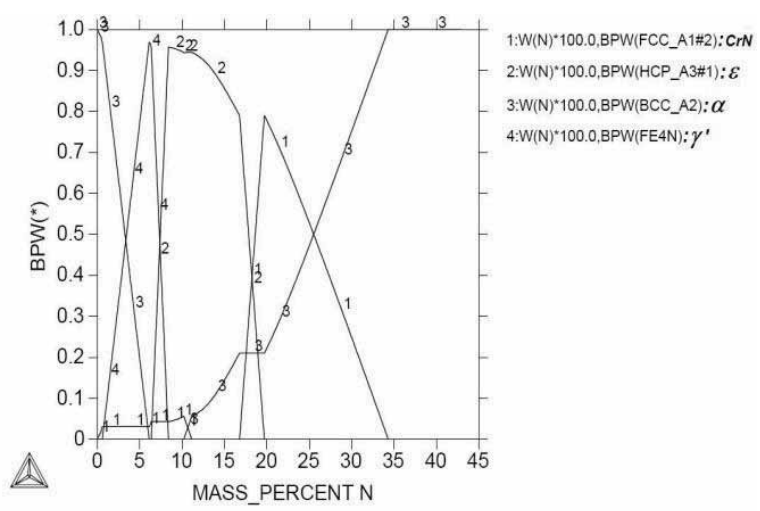

Figure 13. Profil de distribution des phases à $520^{\circ} \mathrm{C}$ et $1 \%$ Cr.

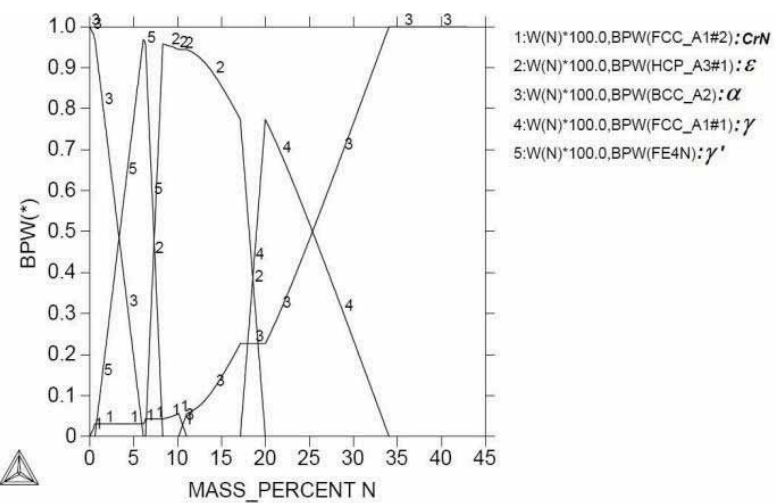

Figure 14. Profil de distribution des phases à $530^{\circ} \mathrm{C}$ et $1 \%$ Cr.

\subsection{Interprétations :}

Si on observe la distribution des phases donnée par le calcul (figures 5 à 7 ) elle est exactement identique à celle obtenue par la caractérisation de nos échantillons (figures, 1 à 3). En augmentant le pourcentage d'azote les phases, $\alpha, \gamma^{\prime}$ et $\varepsilon$, apparaissent respectivement, ce qui correspond au sens allant des couches profondes vers la surface de l'échantillon.

On constate que l'augmentation du pourcentage de chrome augmente la fraction massique du $\mathrm{CrN}$ qui passe de $5.8 \%$ pour $1 \% \mathrm{Cr}$ à $16 \%$ pour $3 \% \mathrm{Cr}$ et qui va jusqu'à $30 \%$ pour $5 \% \mathrm{Cr}$.

La présence du nitrure CrN qui s'étale jusqu'à la couche de combinaison n'est pas fréquemment rencontrée dans les couches nitrurées. Ceci peut être expliqué par la forme de ce nitrure dans ces couches et sa distribution qui font qu'il soit difficilement détectable. Comme ça peut être dû à la mobilité réduite du chrome qui empêche toutes précipitations dans ces phases ce qui donne une microstructure loin de l'équilibre.

Le $\mathrm{Cr}_{2} \mathrm{~N}$ est un nitrure hexagonal pratiquement jamais rencontré dans la nitruration à moyenne température et dans la nitruration classique. La structure d'équilibre calculée montre que ce nitrure est présent lorsque le pourcentage de chrome est élevé (figures 6 à 7). Ceci on ne l'observe que dans le cas de la nitruration des aciers inox contenants de grands pourcentages de chrome. Ou bien dans le cas de la nitruration à hautes températures [10]. D'après nos calculs la phase alpha peut précipiter le nitrure $\mathrm{Cr}_{2} \mathrm{~N}$ dans les conditions où le pourcentage de chrome dépasse les $3 \%$ mais cette précipitation reste faible (1.5\%) (Figure 6) et devient plus abondante à 5 $\% \mathrm{Cr}$ elle est autours de $6 \%$ (figure 7 ). 
Une anomalie est enregistrée dans le cas de la figure 13 ou le ThermoCalc remplace la phase gamma des figures 11 et 12 par le $\mathrm{CrN}$.

Sur les figures 12,13 et 14 nous avons gardé les mêmes paramètres et nous avons changé que la température, on constate sur les figures en question que la légère augmentation de la température n'influe pas sur le pourcentage des phases, mais elle influe sur la distribution de ces phases en fonction du pourcentage d'azote et leur distribution dans la profondeur nitrurée.

Les phases sont légèrement plus condensées dans un domaine de plus en plus restreint en thermes de teneur en azote quand la température augmente. A titre d'exemple le domaine du nitrure $\varepsilon$ s'élargit (présent jusqu'à $20.1 \% \mathrm{~N} / 530^{\circ} \mathrm{C}$ contre $19.2 \% \mathrm{~N} / 510^{\circ} \mathrm{C}$ ).

Si on regarde le profile de concentration d'azote de la figure 9 , on constate que le chrome réduit la solubilité de l'azote dans la phase $\varepsilon$ et l'augmente dans la phase $\mathrm{CrN}$. Et l'augmentation de la quantité d'azote (figure 11) réduit la quantité de chrome dans pratiquement toutes les phases. Le chrome dégagé par les phases favorise la précipitation du nitrure $\mathrm{CrN}$ où l'interaction azote-chrome est la plus favorable.

En générale les cas traités représentent bien les cas retrouvés dans la pratique de la nitruration. Exception faite pour les pourcentages d'azote supérieurs à $15 \%$ où des anomalies sont enregistrées.

On peut dire que la microstructure obtenue après nitruration est proche de l'équilibre, ainsi des calculs thermodynamiques peuvent être utilisés pour extrapoler et explorer d'autres conditions opératoires non encore investies. Ces résultats peuvent être étalés aux cas des aciers $\mathrm{CrMoV}$. On pourra dans ce cas étudier l'influence de la variation des éléments un par un sur la stabilité des phases présentes.

L'intérêt de l'étude thermodynamique se résume à la démonstration que la microstructure obtenue par les calculs est proche des résultats obtenus par l'expérimental. Comme elle permet un accès facile à la distribution des éléments entre les phases.

\section{CONCLUSION}

La présente étude a permis de reprendre le comportement du chrome et de l'azote dans le cas de la nitruration elle et a permis d'exposer l'évolution théorique et pratique des phases nitrures en fonction de la teneur en azote, en chrome et à différentes températures.

Le pourcentage de chrome fusionne les couches nitrures entre elles dans un domaine de précipitation réduit pour aller vers une couche de combinaison faite d'un mélange des phases $\gamma$ ' et $\varepsilon$.

La précipitation des nitrures dans la couche epsilon peut avoir lieu dans les conditions d'équilibre. Car le CrN est stable même dans le domaine de la couche epsilon.

On note que même pour les faibles pourcentages d'azote le nitrure $\mathrm{CrN}$ est présent. Sa présence est due à l'interaction de l'azote avec le chrome dans cette phase et dans les autres phases nitrures qui sont susceptibles de dissoudre cet élément (le chrome). Toutes les phases ont tendance à libérer leur chrome en présence d'azote, sauf pour le cas du nitrure $\mathrm{CrN}$ dont l'augmentation du pourcentage d'azote augmente la solubilité du chrome dans cette phase.

Le $\mathrm{Cr}_{2} \mathrm{~N}$ est thermodynamiquement stable pour des teneurs en chrome qui dépassent les $3 \%$ on est sensé le retrouvé dans la couche de diffusion, mais pratiquement cet équilibre n'est jamais atteint qu'à hautes températures. Ceci s'explique par le changement de structure qu'engendre la formation d'un tel nitrure dans la phase alpha. Ce changement de structure ne peut pas avoir lieu à la température de nitruration à cause de la mobilité réduite du chrome.

Une légère variation de température n'a pas une grande influence sur la distribution des phases dans le cas des faibles pourcentages en chrome

\section{REFERENCES}

[1] J.BARRALIS. Influence des conditions de traitement sur la distribution des phases et des contraintes résiduelles dans les couches nitrurées. Mémoires et Etudes Scientifiques Revue de Métallurgie. Dec, P 629-642. (1987).

[2] C.LEROY. Etude des transformations de phases associées à la nitruration ionique des aciers alliés au chrome. Thèse Nancy I (France) (1983).

[3] M.CONFENTE. Etude physico-chimique et structurale de la nitruration par bombardement ionique à haute intensité des aciers au carbone et aciers alliés. Thèse, Nancy I. (France). (1976).

[4] L.TORCHANE. Control of iron nitride layers growth kinetics in the binary Fe-N system. Metallurgical and materials transactions. V27A, P 1823-1835. (1996).

[5] SOMERS, M.A.J. Layer-growth kinetics on gaseous nitriding of pure iron; evaluation of diffusion coefficients for nitrogen in iron nitrides. Metallurgical and materials transactions.V26A, P 57-74, (1995).

[6] SOMERS, M.A.J.. Precipitation of nitrides in ferritic iron alloys containing chromium. Scandinavian Journal of Metallurgy. №1, P 203-209. (1972).

[7] S.MRIDHA. Metal science. characterization of a nitrided 3\% Chromium steel. Vol16, P 398-404. (1982).

[8] M.POPE. Nitride precipitation in ferritic iron-vanadium alloys. Scandinavian Journal of Metallurgy. $\mathrm{N}^{\circ} 2, \mathrm{P} 29-34$. (1973).

[9] J.H.DRIVER. The electron and field ion metallography of zones in nitrided Fe-Mo alloys. Acta metallurgica. V21, P 1139-1149. (1973).

[10] J.H. SUNG. Phase changes of the AISI 430 ferritic stainless steels after high-temperature gas nitriding and tempering heat treatment. Materials Science and Engineering. A 489 (2008) 38-43 